\title{
Kaposi's disease due to HIV/viral hepatitis \\ B co-infection associated with a Lutz-Lewandowski epidermodysplasia verruciformis in a child in Thiès (Senegal)
}

\section{Gueye Amadou Makhtar', Dioussé Pauline', Bammo Mariama', Dial Chérif', Diallo Thierno Abdoul Aziz', Dione Haby', Seck Fatou', Diallo Ngoné ${ }^{3}$, Fall Codou ${ }^{3}$, Diagne Gueye Ndèye Ramatoulaye ${ }^{1}$, Dieng MameThierno ${ }^{4}$, Diop Bernard Marcel ${ }^{1}$, Ka Mamadou Mourtalla ${ }^{1}$}

${ }^{1}$ Training and Research Unit in Health Sciences, University of Thiès, Senegal, ${ }^{2}$ Department of Anatomical Pathology, Grand Yoff General Hospital, Dakar, Senegal, ${ }^{3}$ Department of Pediatrics, Thiès Regional Hospital, Senegal, ${ }^{4}$ Department of Dermatology, Cheikh Anta Diop University, Dakar, Senegal

Corresponding author: Dr. Amadou Makhtar Gueye, E-mail: gueye_moctar@yahoo.fr

\begin{abstract}
Kaposi's disease is rare in children. We report a mucocutaneous form revealing a co-infection HIV-viral hepatitis B associated with a Lutz-Lewandowski verruciform epidermodysplasia on sickle cell AC. This is a 14 year old boy received in a context of general deterioration. He had angiomatous plaques on the face, the mucous surface of the upper eyelid of the left eye, the trunk, the back and the limbs. His face had pityriasiform, finely scaly papular lesions suggestive of Lutz-Lewandowski's verruciform epidermodysplasia. Complementary examinations showed hypochromic microcytic anemia, positive retroviral serology and Hbs antigen. He was sickle cell type AC. Both HIV serology and Hbs antigen testing were positive in the mother. He was put on triple antiretroviral therapy and the course was unfavorable with death after 3 weeks of hospitalization. Our observation describes a rare case of childhood Kaposi's disease, revealing HIV-viral Hepatitis B co-infection associated with Lutz-Lewandowski's verruciform epidermodysplasia on AC sickle cell.
\end{abstract}

Key words: Kaposi; Verruciform epidermodysplasia; HIV; Hepatitis B; Senegal

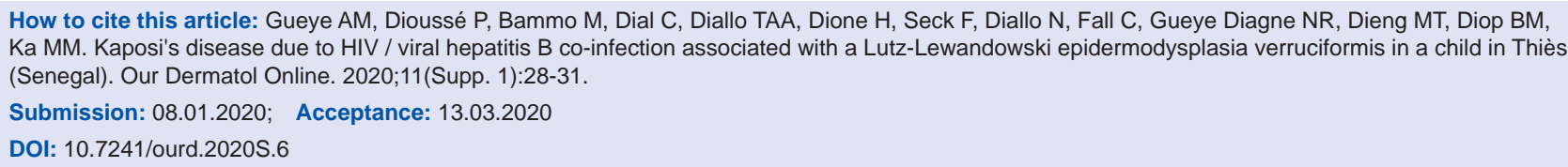




\section{La Maladie de Kaposi sur co-infection VIH/ Hépatite virale B associée à une épidermodysplasie verruciforme de Lutz-Lewandowski chez un enfant à Thiès (Senegal)}

\section{Gueye Amadou Makhtar'1, Dioussé Pauline', Bammo Mariama1, Dial Chérif², Diallo Thierno Abdoul Aziz', Dione Haby', Seck Fatou', Diallo Ngoné ${ }^{3}$, Fall Codou ${ }^{3}$, Diagne Gueye Ndèye Ramatoulaye ${ }^{1}$, Dieng MameThierno ${ }^{4}$, Diop Bernard Marcel', Ka Mamadou Mourtalla ${ }^{1}$}

${ }^{1}$ Training and Research Unit in Health Sciences, University of Thiès, Senegal, ${ }^{2}$ Department of Anatomical Pathology, Grand Yoff General Hospital, Dakar, Senegal, ${ }^{3}$ Department of Pediatrics, Thiès Regional Hospital, Senegal, ${ }^{4}$ Department of Dermatology, Cheikh Anta Diop University, Dakar, Senegal

Corresponding author: Dr. Amadou Makhtar Gueye, E-mail: gueye_moctar@yahoo.fr

\section{RÉSUMÉ}

La maladie de Kaposi est rare chez l'enfant. Nous en rapportons une forme cutanéomuqueuse associée à une coinfection VIH-hépatite virale B et une épidermodysplasie verruciforme de Lutz-Lewandowski sur drépanocytose AC. C'est un garçon de 14 ans reçu pour une altération de l'état général. Il présentait des plaques angiomateuses sur le visage, la muqueuse de la paupière supérieure de l'œil gauche, le tronc, le dos et les membres. Son visage présentait des papules diffuses squameuses pityriasiformes évoquant une épidermodysplasie verruciforme de LutzLewandowski. Les examens complémentaires montraient une anémie hypochrome microcytaire, une sérologie au VIH et l'antigène Hbs positifs. Il était drépanocytaire AC. La sérologie VIH et l'antigène Hbs étaient positifs chez la mère. Il a été mis sous trithérapie antirétrovirale et l'évolution était défavorable avec décès au bout de 3 semaines d'hospitalisation. Notre observation décrit un cas rare de maladie de kaposi de l'enfant, révélant une co-infection VIH-Hépatite virale B associée à une épidermodysplasie verruciforme de Lutz-Lewandowski sur drépanocytose AC.

Mots clés: Kaposi; Epidermodysplasie verruciforme, VIH, Hépatite B, Sénégal

\section{INTRODUCTION}

La maladie de Kaposi est une affection opportuniste fréquente au cours du VIH/SIDA. Elle est couramment rapportée chez l'adulte et rare chez l'enfant [1]. Des cas ont été décrits de manière isolée ou en séries [2-4]. Nous en rapportons un cas de maladie de Kaposi cutanéomuqueux révélateur d'une co-infection VIH-hépatite virale B sur terrain de trait drépanocytaire.

\section{OBSERVATION}

Il s'agit d'un garçon âgé de 14 ans, qui a consulté pour des douleurs abdominales accompagnés de vomissements évoluant depuis 1 mois avec une éruption cutanée, dans un contexte d'altération de l'état général. Linterrogatoire notait une notion de fièvre vespéro-nocturne sans toux ni diarrhée. Sa vaccination était complète selon le programme élargi de vaccination du Sénégal. Son développement psychomoteur est normal. Le poids était

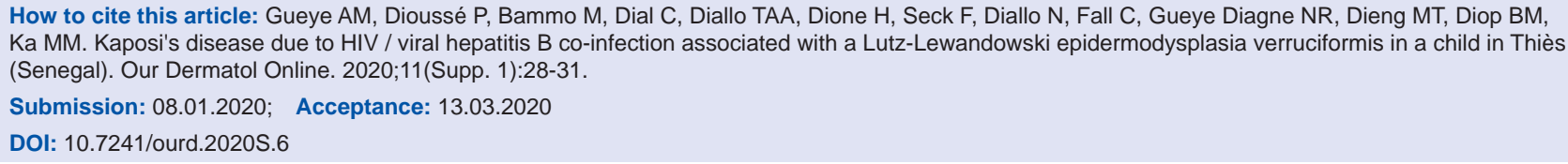


à 28,400kg pour une taille de $160 \mathrm{~cm}(\mathrm{IMC}=11,09)$. Il avait un pli de dénutrition. Lexamen révélait une éruption faite de plaques angiomateuses localisées sur le visage, à la face muqueuse de la paupière supérieur de l'œil gauche, le tronc, le dos et les membres (Fig. 1). Il y avait sur le visage et le décolleté, des lésions papuleuses diffuses finement squameuses pityriasiformes évoquant une épidermodysplasie verruciforme (EDV) de Lutz Lewandowski (Fig. 2). On notait aussi une candidose buccale. Les examens complémentaires avaient montré une anémie hypochrome microcytaire avec un taux d'hémoglobine à $7,9 \mathrm{~g} / \mathrm{dl}$. La sérologie rétrovirale demandée au cours de l'hospitalisation, était revenue positive VIHl. La charge virale était de 4508 copies $/ \mathrm{mm}^{3}$. Lantigène Hbs était positif et il était drépanocytaire type AC. Les transaminases étaient normales. L'échographie abdominale était normale. La radio des poumons était normale.

Lintradermoréaction à la tuberculine était négative de même que la recherche de BK dans les BAAR.

Lexamen anatomopathologique avait confirmé le diagnostic de maladie de Kaposi (Fig. 3). Pour des raisons financières, l'analyse virale et génétique des lésions d'EDV n'a pas été effectuée de même que les autres marqueurs hépatiques La sérologie VIH et de l'antigène Hbs de sa mère étaient positifs, elle n'avait pas de lésions cutanées. Comme traitement il a été transfusé, Fluconazole, ATPE (Aliment thérapeutique prêt à l'emploi), Fer, Albendazole et ARV (Abacavir, Lamivudine et Efavirenz). Lévolution était défavorable, avec décès au bout de 3 semaines d'hospitalisation.

\section{DISCUSSION}

Nous avons rapporté un enfant présentant une maladie de kaposi cutanéomuqueuse, une épidermodysplasie verruciforme de Lutz Lewandowski sur terrain de coinfection VIH-hépatite B et de drépanocytose AC.

La maladie de kaposi sur VIH est rare chez l'enfant. Saka et al a trouvé une proportion $0,2 \%$ chez les enfants de moins de 15 ans [1]. Elle constitue une affection opportuniste fréquente et profuse au cours du VIH/ SIDA, et il a été démontré qu'elle est due au virus herpès humain 8 (HHV8) [5]. Une étude a trouvé que la séroprévalence du kaposi associé au HHV8 était de 7,5\% à $9,0 \%$ chez les enfants sud-africains, elle est passée de $10 \%$ chez les enfants de 2 ans à $30,6 \%$ chez les enfants de 8 ans chez les enfants ougandais [6]. Les modes de transmission varient en fonction des continents. En Afrique, en décrivant des cas familiaux de séropositivité (C) Our Dermatol Online Supp. 1.2020

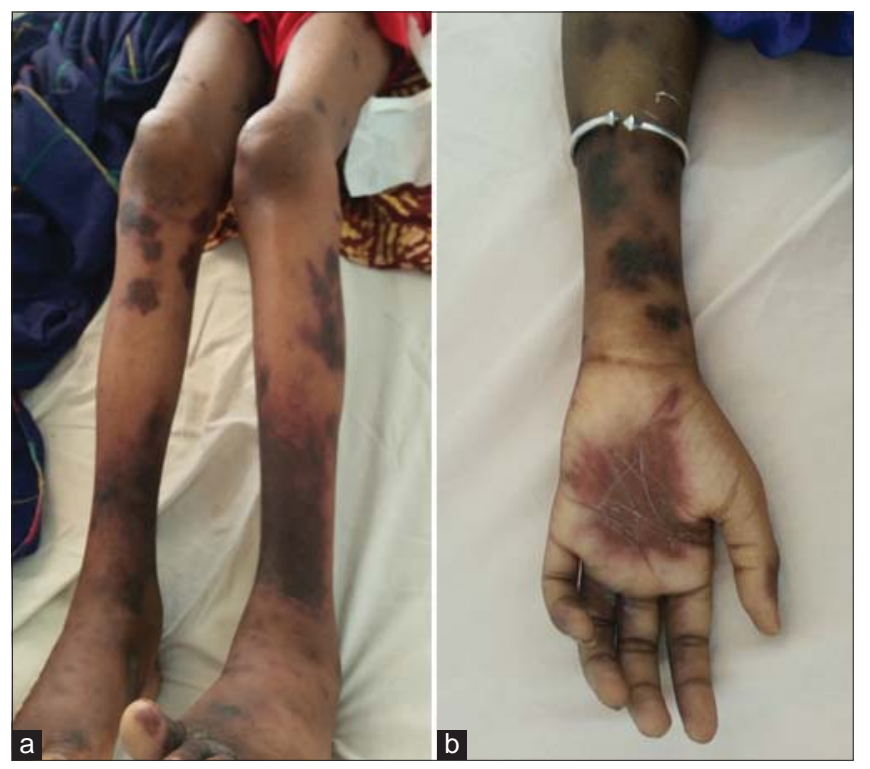

Figura 1: (a) Plaques angiomateuses aux jambes, et (b) au membre supérieur.

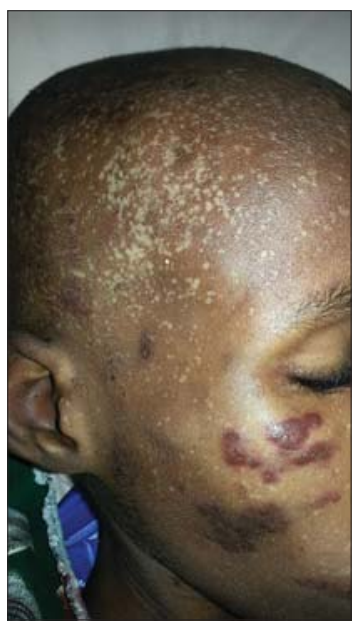

Figura 2: Papules pityriasis versicolor like avec des plaques angiomateuses du visage.

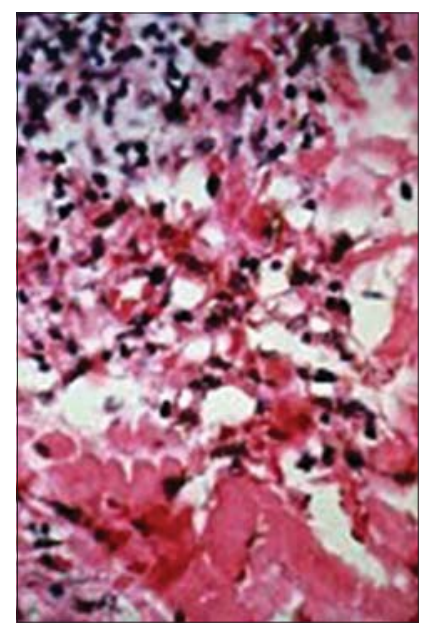

Figura 3: Coupe histologique de Sarcome de Kaposi (forme débutante): on observe quelques foyers de cellules fusiformes, des néo vaisseaux mais surtout un infiltrat inflammatoire lymphoplasmocytaire. 
HHV8, des auteurs ont suggéré que la transmission se faisait de la mère à l'enfant [7].

Chez notre patient la maladie de kaposi avait révélé une co-infection VIH-hépatite B. Au Sénégal, l'hépatite virale $B$ est contractée à la petite enfance. Sa prévalence globale chez les enfants de moins de 5 ans au Sénégal est élevée $(59,38 \%)$ avec la prédominance des formes chroniques [8]. D'où la nécessité d'une vaccination de cette tranche d'âge car ces enfants risquent de devenir des porteurs chroniques.

Quant à l'épidermodysplasie verruciforme, elle a été rapportée chez un enfant infecté par le VIH et présentant une maladie de kaposi [2]. C'est une génodermatose à transmission autosomique récessive, due à l'infection par des papillomavirus. Les lésions cutanées apparaissent dès le plus jeune âge, généralement au cours de la première décennie et persistent. Deux locus de prédisposition ont été identifiés sur le chromosome 17 et le chromosome $2[9,10]$.

Notre enfant est porteur de trait drépanocytaire; la drépanocytose se définit comme une maladie héréditaire à transmission autosomique récessive caractérisée par la présence de l'hémoglobine $\mathrm{S}$ dans les hématies, responsable de leur déformation en faucille en cas d'hypoxie. Au Sénégal, il existe une nette amélioration de la sécurité transfusionnelle avec une réduction du taux de la transmission sanguine du VIH et l'hépatite virale B [11].

Notre patient présente la forme hétérozygote qui est typiquement asymptomatique sur le plan clinique. Bien qu'il n'ait jamais reçu une transfusion sanguine avant son hospitalisation, il présente plusieurs facteurs de survenue de l'anémie. Létude de Goby et al. a montré que le niveau de contamination par le VIH et l'hépatite virale B était plus élevé chez les enfants drépanocytaires SS ayant reçu plus de trois transfusions [12].

\section{CONCLUSION}

Notre observation décrit un cas rare de maladie de kaposi de l'enfant, révélant une coinfection VIH-hépatite virale B sur terrain de trait drépanocytaire. Les infections virales concomitantes à l'infection VIH et le retard du diagnostic et de la prise en charge ont assombri le pronostic de la maladie dont l'évolution a été fatale.

\section{Consent}

The examination of the patient was conducted according to the Declaration of Helsinki principles.
The authors certify that they have obtained all appropriate patient consent forms. In the form the patient(s) has/have given his/her/their consent for his/her/their images and other clinical information to be reported in the journal. The patients understand that their names and initials will not be published and due efforts will be made to conceal their identity, but anonymity cannot be guaranteed.

\section{REFERENCES}

1. Saka B, Mouhari-Toure A, Wateba IM, Akakpo S, Kombaté K, Balaka A, et al. Maladie de Kaposi associée au VIH: 103 observations en dermatologie à Lomé (Togo). Méd Santé Trop. 2013;23:109-11.

2. Dicko A, Faye O, Traoré A, Keita H, Tall K, Guindo B, et al. Maladie de Kaposi profuse chez un enfant VIH positif. Ann Dermatol Vénéréologie. 2015;142:699.

3. Saka B, Souley Z, Kombaté K, Mouhari-Toure A, Akakpo S, NapoKoura $\mathrm{G}$, et al. Les cancers cutanés au Togo: 223 observations. Med Trop. 2010; 70:169-71.

4. Asuquo ME, Ebughe G. Previous article in issue: Major dermatological malignancies encountered in the University of Calabar Teaching Hospital, Calabar, southern Nigeria. Int J Dermat. 2012;51:36-40.

5. Katano H, Iwasaki T, Baba N, Terai M, Mori S, Iwamoto A, et al. Identification of antigenic proteins encoded by human herpesvirus 8 and seroprevalence in the general population and among patients with and without Kaposi's sarcoma. J Virol. 2000;74:3478-85.

6. Butler LM, Dorsey G, Hladik W, Rosenthal PJ, Brander C, Neilands TB, et al. Kaposi sarcoma-associated herpes virus seroprevalence in population-based samples of african children: evidence for at least 2 patterns of Kaposi sarcoma-associated herpes virus transmission. J Infect Dis. 2009;200:430-8.

7. Charfi S, Chaabane H, Chaari I, Kallel R, Turki H, Boudawara TS. De novo anaplastic Kaposi sarcoma in a HIV-negative man: A case report and review of the literature. Our Dermatol Online. 2017;8:296-9.

8. Sall Diallo A, Sarr M, Fall Y, Diagne C, Kane MO. Etude de l'infection par le virus de l'hépatite virale $\mathrm{B}$ dans la population infantile au Sénégal. Dakar Med. 2004; 49:136-42.

9. Rodríguez L, Contreras R, Di Martino Ortiz B, de Lezcano LB. Acquired epidermodysplasia verruciformis i $\mathrm{n}$ an HIV positive child. Report of a case. Our Dermatol Online. 2015;6:32-5.

10. Brzezinski P, Martini L. Usage of unsaturated esters of retinol to defeat Hopf's acrokeratosis verruciformis in hands and feet of a young man. Our Dermatol Online. 2018;9:339-40.

11. Touré-Fall AO, Dièye TND, Sall A, Diop M, Seck M, Diop S, et al. Risque résiduel de transmission du VIH et du VHB par transfusion sanguine entre 2003 et 2005 au Centre national de transfusion sanguine de Dakar (Sénégal). Trans Clin Biol. 2009;16:439-43.

12. Gody JC, Essomo Megnier-Mbo CM, Chelo D, Guindo A, Gabato W, Bureau JJ, et al. Survenue du VIH et du VHB dans une Cohorte d'Enfants Drépanocytaires Transfusés au Complexe Pédiatrique de Bangui. Health Sci Dis. 2014;15:1.

Copyright by Amadou Makhtar Gueye, et al. This is an open-access article distributed under the terms of the Creative Commons Attribution License, which permits unrestricted use, distribution, and reproduction in any medium, provided the original author and source are credited. Source of Support: We could not have benefited from support for the writing of this article, Conflict of Interest: We did not encounter any conflict of interest in the writing of this article. 